Conclusions These data (Cui, et al. PLoS One 2018;13: e01931711), also show strong association of EBV with SLE with anti-EBNA1 being more frequent in SLE cases than in concurrent patient controls, supporting the known association of EBV infection with SLE. Further, anti-EBNA1 is virtually always present in SLE and is present more frequently in EBA-infected SLE patients than in EBV-infected controls, consistent with the hypothesis that anti-EBNA1 is the usual immune response foundation from which pathogenic SLE autoimmunity emerges. These results support a model of the etiology of SLE that begins with EBV infection, leading to anti-EBNA1 antibodies. Then, a subset of these heteroimmune anti-EBNA1 antibodies cross react with SLE autoantigens (e.g., $\mathrm{Sm}$ as in figure 1, (EBV image, courtesy of NIAID/NIH; SLE malar rash image, courtesy of Mayo Foundation, all rights reserved)) that then mature into an SLE autoantibody response and result in in life-threatening clinical SLE. The specific role of the other EBV gene products, EBNA2, EBNA3C, and EBNALP, in this process (figure 1) await elucidation.

Acknowledgments Support is appreciated from US Department of Veterans Affairs Merit Award (I01 BX001834), and the National Institutes of Health (R01 AI24717 \& $\quad$ U01 AI130830).

\section{ANTI-RNP ANTIBODIES ARE ASSOCIATED WITH THE INTERFERON GENE SIGNATURE BUT NOT COMPLEMENT ACTIVATION IN SLE}

${ }^{1}$ Erika Hubbard*, ${ }^{2,3}$ David S Pisetsky, ${ }^{1}$ Peter Lipsky. 'AMPEL BioSolutions, LLC and RILITE Foundation, Charlottesville, VA, USA; ${ }^{2}$ Duke University Medical Center, Durham, NC, USA; ${ }^{3}$ VA Medical Center, Durham, NC, USA

10.1136/lupus-2021-lupus21century. 102

Background Anti-nuclear antibodies are essential features of SLE and are important markers for both diagnosis and pathogenesis. Anti-double stranded DNA (dsDNA) antibodies, which are routinely monitored to assess disease activity, can form immune complexes that activate complement (C) and promote renal pathology. Relatively less is known about the roles of autoantibodies against RNA binding proteins (RBPs) in pathogenesis and about the relationship between antidsDNA, anti-RBPs, complement activation, and expression of the interferon (IFN) gene signature (IGS). Analysis of data from two clinical trials of tabalumab in SLE (Illuminate $1 \&$ 2, GSE88884) was undertaken to understand these interrelationships.

Methods Microarray data from 1620 active (SLEDAI $\geq 6$ ), female SLE patients and accompanying laboratory measurements were analyzed. Gene set variation analysis (GSVA) determined enrichment of transcriptomic signatures in each patient. Linear regression analysis was used to determine relationships between IGS GSVA scores and C3 and/or C4 levels as well as autoantibody levels. Unbiased classification and regression trees (CART) were constructed to determine the highest predictors of IGS expression.

Results Patients were initially stratified by autoantibody positivity. Comparison of GSVA enrichment scores of a common IGS demonstrated that SLE patients positive for anti-RNP antibodies alone had greater enrichment of the IGS than those positive for anti-dsDNA alone. Similar results were noted for gene signatures of type I IFN, IFN $\alpha 2$, IFN $\beta$, and IFN $\gamma$. In contrast, the TNF-induced gene signature was observed comparably in patients with anti-dsDNA or anti-RNP antibodies and an IL-1 gene signature was only observed in those with anti-dsDNA. By linear regression, IFN GSVA scores and C3 and C4 levels were significantly, inversely related in antidsDNA+ patients but not those with anti-RNP antibodies. Antibody levels correlated with decreased C levels in dsDNA+ patients. Additionally, IGS GSVA scores were increased in anti-dsDNA+ patients with low C3 or C4 compared to antidsDNA+ patients with high/normal C, but there was no significant difference in IGS expression in anti-RNP+ patients with the same stratification. CART analysis identified anti-RNP status as the highest predictor of the IGS GSVA score. Finally, $54.5 \%$ of SLE patients without IGS expression exhibited autoantibodies, but only $13.7 \%$ of SLE patients negative for autoantibodies expressed the IGS signature.

Conclusions Taken together, these data indicate that anti-RNP antibodies are associated with IGS expression more strongly than anti-dsDNA antibodies but are not related to the depression of C3 or C4. Furthermore, IGS expression is not required for autoantibody production, but autoantibodies are likely directionally related to the IGS signature.

\section{PRELIMINARY DATA ON THE MAPPING OF ANTI- MITOCHONDRIAL ANTIBODIES IN SYSTEMIC LUPUS ERYTHEMATOSUS}

\begin{abstract}
1,2 Yann LC Becker*, 1,2 Emmanuelle Rollet-Labelle, ${ }^{1,2}$ Tania Lévesque, ${ }^{1,2}$ Joannie Leclerc, ${ }^{3}$ Anne-Sophie Julien, ${ }^{1,4}$ Paul R Fortin, SLICC inception cohort investigators, 1,2Éric Boilard. ${ }^{1}$ Centre de Recherche ARThrite - Arthrite, Recherche et Traitements, Université Laval, Québec, QC, Canada; ${ }^{2}$ Centre de Recherche du CHU de Québec - Université Laval, Québec, QC, Canada; ${ }^{3}$ Département de mathématiques et statistique, Université Laval, Québec, QC, Canada; ${ }^{4}$ Division de Rhumatologie, Département de Médecine, CHU de Québec Université Laval, Québec, QC, Canada
\end{abstract}

10.1136/lupus-2021-lupus21 century. 103

Background In systemic lupus erythematosus (SLE), mitochondria and their inner components may be released into the extracellular space, potentially eliciting a pro-inflammatory response by the immune system. While cardiolipin was long known as a mitochondrial target of autoantibodies, we reported in previous case-control studies that autoantibodies to whole mitochondria (AwMA), mitochondrial DNA (AmtDNA) and mitochondrial RNA (AmtRNA) are also targeted by SLE autoantibodies. We aim to characterize levels of these autoantibodies throughout SLE disease progression.

Methods Anti-mitochondrial antibodies (AMA, IgGs) targeting whole mitochondria (AwMA), mtDNA (AmtDNA) or mtRNA (AmtRNA) were measured by direct-ELISA, using sera from the Systemic Lupus International Collaborating Clinics (SLICC) inception cohort. Samples comprised healthy controls $(n=127)$ and 3453 samples obtained from 816 SLE patients, between the diagnosis up to 7 years afterward. Institution of the SLICC cohort obtained approval from their local research ethic boards and written consent from every participant. Eligibility to the cohort, within 15 months of diagnosis, was conditional to the positivity to 4 , or more, ACR criteria for the classification of SLE. AMA levels are expressed as the median optical density measured at $405 \mathrm{~nm} \pm$ interquartile range. Differences in AMA levels between healthy donors and baseline SLE samples were assessed, using Mann-Whitney tests and 
Spearman tests were used to assess correlations between levels of the various AMA.

Results Preliminary results indicate that, among the various subsets of IgGs targeting mitochondrial components, AwMA and AmtRNA but not AmtDNA were significantly increased in newly diagnosed SLE patients, in comparison with healthy individuals (respectively: $\mathrm{p}=0.004, \mathrm{p}<0.0001$, and $\mathrm{p}=0.1$. figure 1). While AwMA levels remain constant for two years into the disease (Enrollment: 0.11 \pm 1.37 ), an increase is observed after the third year (year 3: $0.17 \pm 0.67$ ). A similar effect is measured for AmtRNA levels (Enrollment: 0.21 \pm 2.46 . Year 3: 0.36 \pm 1.81 ). AwMA levels are correlated to those of AmtDNA and AmtRNA $\left(p<0.0001\right.$. Respectively $r_{s}=0.32$ and $\left.r_{s}=0.38\right)$ and levels of both anti-mitochondrial nucleic acids displayed stronger correlations $\left(\mathrm{p}<0.0001 ; \mathrm{r}_{\mathrm{s}}=0.55\right)$.
Conclusions Levels of circulating autoantibodies to whole mitochondria and mtRNA at baseline allow discriminating between healthy individuals and SLE patients. While levels of AMA appear to be fluctuating throughout the disease, further biostatistical analyses will be performed to assess associations between AMA and clinical manifestations and outcomes of the disease. These data will allow to appreciate the quality of AMA as biomarkers in the prediction of clinical outcomes, damages, or the clustering of patients in SLE.

Acknowledgments Dr. Paul R. Fortin's (PRF) work is supported by a Canada Research Chair. This study was supported by Canadian Institutes of Health Research (CIHR) grants to PF and Dr. Éric Boilard (EB). Yann LC Becker and EB are recipients of awards from the Fond de Recherche en Santé $d u$ Québec (FRSQ).
A.

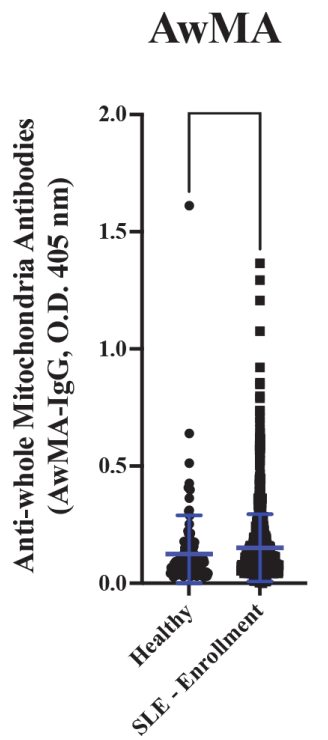

B.

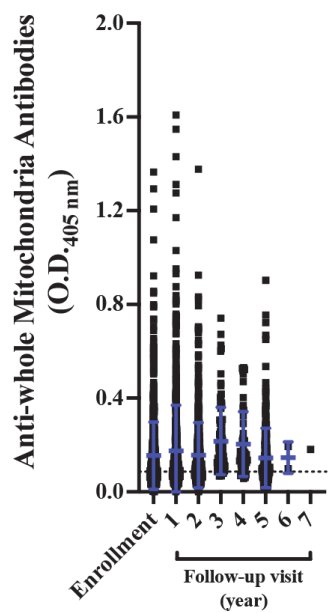

AmtDNA
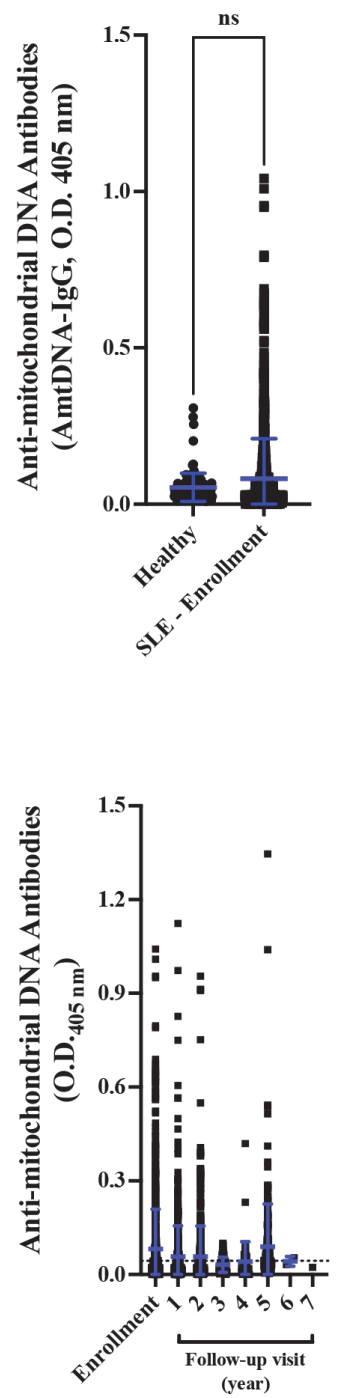

AmtRNA
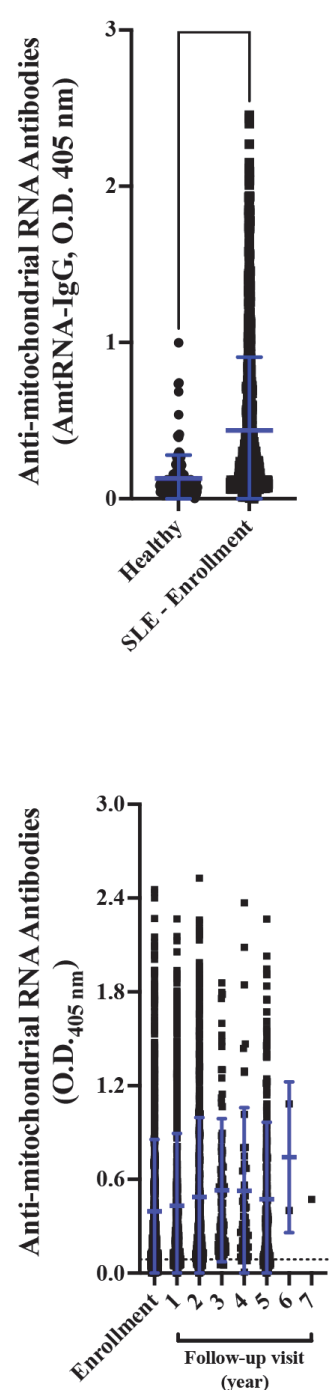

Abstract 1708 Figure 1 Levels of circulating IgG against whole mitochondria (AwMA), mitochondrial DNA (AmtDNA) or RNA (AmtRNA) were assessed by direct ELISA. (A) AwMA and AmtRNA but not AmtDNA were significantly increased in newly diagnosed SLE patients (i.e. enrollment), compared to healthy controls. Mann-Whitney test. Data are median \pm standard deviation (SD). Ns: not significant $(p>0.05)$. ${ }^{* * *} p<0.001$. ${ }^{* * * *} p<$ 0.0001 . (B) Levels of AMA were assessed at various time points during the disease for subsequent biostatistical analyses. Data are median \pm SD. The dotted line represents the median value for the healthy group. 